\title{
Hemodynamic variation is a dominant contributing factor of Graves' hyperthyroidism complication: Heart failure and fatal liver disfunction: a case report and analysis
}

\author{
Yi Fang ${ }^{1}$, Zhiyuan $\mathrm{Li}^{1}$, Jinjing Wang ${ }^{1}$, Jianbin $\mathrm{Su}^{1}$, Jing Zeng ${ }^{1}$, Jian $\mathrm{Xue}^{1}$, and Xinyu $\mathrm{Li}^{2}$ \\ ${ }^{1} 5$ th Medical Center of Chinese PLA General Hospital \\ ${ }^{2}$ Capital Medical University
}

September 25, 2021

\begin{abstract}
Tachycardia and atrial fibrillation are cardiovascular symptoms of Graves' hyperthyroidism, if left untreated and further deterioration can result in heart failure and liver dysfunction, we describe a female patient to present the continuum of the pathophysiology development, to highlight the hemodynamic variation is dominant contributing factor of Graves' hyperthyroidism complication
\end{abstract}

\section{Introduction}

Graves' hyperthyroidism is an autoimmune disease resulting in excessive endogenous thyroid hormone production and release ${ }^{1}$, which is associated with multisystem involvement. Graves' hyperthyroidism presents with the features of palpitation, dyspnea, weight loss, tremors, and nausea. More evidences suggest cardiovascular complications account for most of the deaths in hyperthyroid patient ${ }^{8}$, it have significant effects on cardiovascular system through direct and indirect mechanisms ${ }^{2}$. We describe a long history young female patient of Graves' hyperthyroidism with tachycardia, atrial fibrillation, congestive heart failure, severe hepatic dysfunction, through this case report, we focus on the role of hemodynamics in severe complications of hyperthyroidism, analyzed with review literature and present effective treatment strategies as well.

\section{Case report}

A 37-year-old female with intermittent palpitations, easy fatigability for 18 years was referred to our hospital with 6 weeks of exertional dyspnea, lower extremity edema. She was diagnosed with Graves' hyperthyroidism in 2001 and started on methimazole (MMI), but compliance taking the medication was low. She had taken herbal remedies for two years, but the detailed prescription cannot be provided. She admitted and received medical treatment at local hospital 4 weeks ago, however, her condition deteriorated and total bilirubin is about five times higher than normal. Then, she was referred to our hospital. On physical examination, she was afebrile, heart beats at presentation was 105 per minute and blood pressure was $116 / 65 \mathrm{mmHg}$. She had mild generalized icterus but no consciousness disturbance,diffusely enlarged thyroid gland without pain. Two red spots on the upper chest were noticed that characterized by central arteriole with radiated blood vessels. Cardiovascular exam revealed an irregular rhythm, systolic murmur at the lower left sternal border, the jugular venous pressure was raised and neck veins engorged. Mild bilateral lower extremity edema was present. A workup for thyroid dysfunction demonstrated raised serum free thyroxine, triiodothyronine levels with suppressed thyroid stimulating hormone (TSH) level and high TSH receptor antibody titers (Table 1). Differential complete blood count showed a low white blood cell count and low absolute neutrophil count. Increased BNP indicated congestive heart failure, cardiac biomarkers were negative for an acute coronary 
event. Liver chemistries showed mild elevations in aspartate aminotransferase, $\gamma$-glutamyl transpeptidase and blood ammonia. Notably, her total bilirubin, direct bilirubin elevated progressively and coagulation disorders were confirmed. The patient also had negative serology for hepatitis A, B, and C. Autoimmunity markers (antinuclear antibody, antimitochondrial antibody, antineutrophil cytoplasmic antibody, anti-smooth-muscle antibody, and anti-liver-kidney microsome antibody) were all negative • The ECG showed atrial fibrillation with ventricular rate at 80 beats/min (given metoprolol, $25 \mathrm{mg} 2 / \mathrm{d}$ ), chest radiography showed right pleural effusion, pulmonary congestion and the cardiothoracic ratio was $72 \%$ (Figure 1). Transthoracic echocardiogram reported an ejection fraction $(\mathrm{EF})$ of $60 \%$ with bilateral atrial and right ventricle enlargement, elevated pulmonary artery pressure, severe mitral and tricuspid regurgitation(Figure 2). Abdominal ultrasonography, computed tomography and magnetic resonance cholangiopancreatography showed no biliary ductal dilation, pancreatic, biliary, or intrahepatic mass, portal vein thrombosis, or findings concerning for primary sclerosing cholangitis. Moderate ascites was found in right sided chest cavity,cavum pericardii,abdomen - pelvic cavity $\circ$ A thyroid nuclear scan showed a diffuse homogenous increased uptake of radioactive iodine with no background uptake.

Table 1 Laboratory data on admission.

\begin{tabular}{|c|c|c|c|c|c|}
\hline Peripheral blood & Peripheral blood & Peripheral blood & $\mathrm{Ca}(\mathrm{mmol} / \mathrm{L})$ & 2.24 & $(2.15-2.57)$ \\
\hline $\mathrm{WBC}\left(10^{9} / \mathrm{L}\right)$ & 2.7 & $(3.5-9.5)$ & $\mathrm{BUN}(\mathrm{mmol} / \mathrm{L})$ & 4.58 & $(1.4-8.3)$ \\
\hline $\operatorname{NEUT}\left(10^{9} / \mathrm{L}\right)$ & 1.13 & $(1.8-6.3)$ & $\mathrm{Cre}(\mathrm{mmol} / \mathrm{L})$ & 34 & $(55-110)$ \\
\hline $\operatorname{RBC}\left(10^{12} / \mathrm{L}\right)$ & 3.68 & $(3.8-5.1)$ & $\mathrm{LDH}(\mathrm{U} / \mathrm{L})$ & 198 & $(109-245)$ \\
\hline $\mathrm{Hb}(\mathrm{g} / \mathrm{L})$ & 105 & $(115-150)$ & LDL-C(mmol/L) & 1.36 & $(1.9-3.8)$ \\
\hline Plt $\left(10^{12} / \mathrm{L}\right)$ & 97 & $(125-350)$ & $\mathrm{PG}(\mathrm{mmol} / \mathrm{L})$ & 3.85 & $(3.9-6.1)$ \\
\hline $\mathrm{PT}(\mathrm{s})$ & 20.1 & $(8.8-12.8)$ & $\operatorname{Tn}-\mathrm{I}(\mathrm{ng} / \mathrm{ml})$ & $<0.012$ & $(0-0.16)$ \\
\hline PTA(\%) APTT(sec) & 4745.9 & $(80-150)(24.9-36.8)$ & $\mathrm{CK}-\mathrm{MB}(\mathrm{U} / \mathrm{L}) \mathrm{BNP}(\mathrm{pg} / \mathrm{ml})$ & 221910 & $([?] 25)(0-12$ \\
\hline PT-INR & 1.77 & $(0.8-1.5)$ & $\operatorname{AMON}(\mu \mathrm{mol} / \mathrm{L})$ & 54 & $(9-33)$ \\
\hline Serum & Serum & Serum & $\mathrm{TSH}(\mu \mathrm{IU} / \mathrm{L})$ & $<0.008$ & $(0.55-4.78)$ \\
\hline $\mathrm{TP}(\mathrm{g} / \mathrm{L})$ & 71 & $(65-85)$ & FT3(pmol /L) & 24.1 & $(2.8-6.3)$ \\
\hline $\operatorname{Alb}(g / L)$ & 29 & $(40-55)$ & $\mathrm{FT} 4(\mathrm{pmol} / \mathrm{L})$ & 109.13 & $(11.5-22.7)$ \\
\hline $\operatorname{ALT}(\mathrm{U} / \mathrm{L})$ & 13 & $(7-40)$ & $\operatorname{Trab}(\mathrm{IU} / \mathrm{L})$ & 37.88 & $(<1.75)$ \\
\hline $\operatorname{AST}(\mathrm{U} / \mathrm{L})$ & 56 & $(13-35)$ & Blood gas analysis & (nasal $\mathrm{O}_{2} 3 \mathrm{~L}$ ) & \\
\hline T-Bil $(\mu \mathrm{mol} / \mathrm{L})$ & 106.5 & $(2-20)$ & $\mathrm{pH}$ & 7.435 & (7.350-7.450 \\
\hline D-Bil $(\mu \mathrm{mol} / \mathrm{L})$ & 64.7 & $(0-6.8)$ & $\mathrm{pCO}_{2}(\mathrm{mmHg})$ & 31.3 & $(32-48)$ \\
\hline $\mathrm{TBA}(\mu \mathrm{mol} / \mathrm{L})$ & 19.1 & $(0-15)$ & $\mathrm{pO}_{2}(\mathrm{mmHg})$ & 89.7 & $(80-100)$ \\
\hline $\operatorname{ALP}(U / L)$ & 85 & $(40-150)$ & $\mathrm{HCO}_{3}(\mathrm{mmol} / \mathrm{L})$ & 22.4 & $(21-27)$ \\
\hline $\boldsymbol{\gamma}-\mathrm{GTP}(\mathrm{U} / \mathrm{L})$ & 88 & $(7-45)$ & $\mathrm{BE}(\mathrm{mmol} / \mathrm{L})$ & -2.9 & $(-3-3)$ \\
\hline $\mathrm{K}(\mathrm{mmol} / \mathrm{L})$ & 4.29 & $(3.5-5.3)$ & $\mathrm{Lac}(\mathrm{mmol} / \mathrm{L})$ & 1.9 & $(0.5-1.6)$ \\
\hline $\mathrm{Na}(\mathrm{mmol} / \mathrm{L})$ & 134 & $(137-147)$ & & & \\
\hline $\mathrm{Cl}(\mathrm{mmol} / \mathrm{L})$ & 102 & $(99-110)$ & & & \\
\hline
\end{tabular}

$\boldsymbol{\gamma}$ GTP, $\boldsymbol{\gamma}$-glutamyl transpeptidase; Alb, albumin; ALP, alkaline phosphatase; ALT, alanineaminotransferase; AMON plasma ammonia APTT, activated partial thromboplastin time; AST, aspartate aminotransferase; BE, base excess; BNP, brain natriuretic peptide; BUN, blood urea nitrogen; CK, creatinine kinase; CK-MB, creatinine kinase-muscle-brain isozyme; $\mathrm{Cr}$, creatinine; FT3, free triiodothyronine; FT4, free thyroxine; Hb, hemoglobin; LDL-C, low-density lipoprotein cholesterol LDH, lactate dehydrogenase NEUT neutrophile granulocyte PG, plasma glucose; Plt, platelets; PT, prothrombin time; PTA, Prothrombin activity;PT-INR, prothrombin time international normalized ratio; RBC, red blood cells; TBA, total bile acid, T-Bil, total bilirubin; Tn-I, troponin I; TP, total protein; TRAb, thyrotrophin receptor antibody; TSH, thyroid-stimulating hormone; WBC, white blood cells; Numerals in parentheses are normal values.

She was diagnosed with Graves' hyperthyroidism accompanied by atrial fibrillation,congestive heart failure,Liver function and coagulation function supported the diagnosis of acute-on-chronic pre-liver failure. 
She was continued with furosemide and metoprolol, aspirin was ceased because of hemorrhagic tendency. However, symptoms of atrial fibrillation and heart failure did not improve significantly, BNP continued to rise. The treatment plan switched to methimazole cream $5 \mathrm{mg}(0.1 \mathrm{~g}) 2 /$ day to diminish levels of thyroid hormone, (the concentration-time curves of the novel formulation in a preclinical study demonstrated the concentration of the methimazole cream of neck swearing group was higher than oral group in thyroid gland tissue and action time could be extended by percutaneous administration)bisoprolol $2.5 \mathrm{mg} /$ day to control ventricular rate at $70^{\sim} 90$ beats/min, Torasemide injection at $20 \mathrm{mg} /$ day and spironolactone tablets at $40 \mathrm{mg} /$ day were admitted, maximum dose of torasemide reached at $80 \mathrm{mg} /$ day. Consequently, the body mass steadily decreased to $56 \mathrm{~kg}$, her dyspnea, lower limb edema and other symptoms gradually alleviated. On the 10th day of admission, the electrocardiogram showed that sinus rhythm was restored, and the ventricular rate was maintained at 60 beats/min.

Ademetionine for injection and capsule ursodeoxycholic acid were initialed on the second day of admission. Although her general condition was improving, total bilirubin levels increased progressively (Fig $\mathbf{3}$ ) . She was started on daily therapeutic plasma exchange (TPE) on hospital day 4, replacement fluid contained half fresh frozen plasma (FFP) and half $5 \%$ albumin was equal in volume to her total plasma volume (about $4 \mathrm{~L}$ ). Intravenous methylpredisolone at $40 \mathrm{mg}$ /day was given for treatment of severe hepatic dysfunction, total bilirubin levels peaked at $279.8 \mu \mathrm{mol} / \mathrm{L}$ (direct bilirubin of $177.7 \mu \mathrm{mol} / \mathrm{L}$ ) on hospital day 11 , then the total bilirubin gradually decreased. The serum free triiodothyronine and thyroxine levels were normalized on the twelfth day of admission. She was treated with radioactive iodine at a dose of 50mci as planned after the tenth course of TPE. The patient was discharged home on bisoprolol, oral diuretics, methylpredisolone tablet and capsule ursodeoxycholic acid. By 3 months post discharge, her total bilirubin had also normalized.

\section{Discussion}

Thyroid hormones have significant effects on cardiovascular system through genomic nuclear and extranuclear nongenomic mechanisms, which have been well worked out. Both of them act together to regulate cardiac function and cardiovascular hemodynamics ${ }^{4}$ 。 Excess thyroid hormone acting on the heart and peripheral vasculature include decreased systemic vascular resistance (SVR) and increased resting heart rate, left ventricular contractility, and blood volume ${ }^{5}$. The decreased systemic vascular resistance leads to reduction in renal perfusion pressure, activation of the renin-angiotensin-aldosterone system (RAAS), thereby promotes sodium retention and blood volume in the body ${ }^{6}$. These Hemodynamic variations combine to promote an increase in blood volume and preload, which increase cardiac output $50 \%$ to $300 \%$ higher than in normal individuals ${ }^{79}$. Excess amounts of thyroid hormone have a significant impact on cardiovascular hemodynamics have been observed in our patient, including tachycardia, widened pulse pressure,atrial fibrillation,cardiomegaly, congestive heart failure.

Tachycardia is the most common rhythm disturbance and recorded in almost all patients with hyperthyroidism ${ }^{10}$, Atrial fibrillation occurs in 10 to 25 percent of patients with hyperthyroidism ${ }^{11} 12$. An investigation in Japan revealed that tachycardia [?]150 bpm was associated with increased mortality in thyroid storm patients ${ }^{13}$, The presence of $\mathrm{AF}$ in patients with hyperthyroidism is associated with significant mortality ${ }^{14}$. Increased left ventricular mass caused by elevated left atrial pressure,impaired ventricular relaxation, ischemia resulting from raised resting heart rate and increased atrial ectopic activity ${ }^{15}$, these underlying factors relates to atrial fibrillation accelerate systemic hemodynamic deterioration. More evidence suggest cardiovascular complications account for most of the deaths in hyperthyroid patient ${ }^{8}$. Therefore cardiac manifestations in the setting of thyrotoxicosis should be treated promptly and aggressively. Beta-blockers are selected as first-line treatment for ventricular rate control of Graves' hyperthyroidism. They not only to help ameliorate symptoms such as palpitations and tremor, especially in the stage before ATD take effect. but also to decrease the ventricular response to AF by action on the $\beta 1$ receptors,. Betablockers (such as landiolol, esmolol and bisoprolol) with higher selectivity in cardiovascular system have higher cardioprotective effects and superior prevention of atrial fibrillation, especially for patients with bronchospasm ${ }^{16}$.

Hyperthyroidism may complicate, if left untreated tachycardia and AF last for a long period of time,hemodynamic changes predispose the patient to heart failure. the concept of "tachycardia-induced 
cardiomyopathy" related to hyperthyroidism is more plausible, as HF commonly improves with adequate control of the heart rate and AF when the euthyroid state is restored. Some reported cases of congestive heart failure with Graves' hyperthyroidism had increased pulmonary resistance, autoimmune mechanisms with subsequent endothelial damage may have an important role in its occurrence ${ }^{17} 18$. Our patient had features of high-output congestive heart failure with pulmonary hypertension, as evidenced by raised jugular venous pressure neck veins engorged and bilateral pedal edema, Echocardiogram reported RVDd is increased significantly in our patients,but SV and CO of left ventricle almost normal(Figure 2), which means Graves' hyperthyroidism causes increased cardiac output and a hyperdynamic right ventricle, right ventricular function decreases more significantly than the left ventricular function. The deteriorating of congestive heart failure due to hyperthyroidism in our patient means it is not limited to the elderly, but can develop even in young patients.

Brain natriuretic peptide (BNP) is mainly synthesized and secreted by myocytes in the left ventricle (LV) as a response to myocytes stretched by pressure overload or volume expansion of the ventricle,Although BNP is steadily increased in HF, it may often be insufficient to reduce the sodium retention and vascular constriction due to activation of RAAS ${ }^{19}$. Therefore, use of diuretics is fundamental in the treatment of signs of fluid overload and congestion in patients with HF in hyperthyroidism. Initial treatment with furosemide in our patient is ineffective, torasemide was admitted intravenously, as the clinical trial has suggested patients treated with torasemide fluid overload and symptoms improved more than in the furosemide group. Meanwhile, using the aldosterone receptor antagonist spironolactone to block RAS system, which acts primarily by competitive binding to the aldosterone-dependent sodium-potassium exchange sites located in the DCT and collecting duct. The effect of the blockade is to decrease sodium reabsorption with water retention and to increased potassium retention.

Compare with the increased cardiac output and peripheral circulation, the hepatic blood flow is little increased in the early stage of Graves' hyperthyroidism. Under this condition, the growth of splanchnic oxygen consumption in hyperthyroidism is accomplished by an increased oxygen extraction. This could result in anoxia of the centro-lobular zones of the liver and may well be related to the centro-lobular necrosis ${ }^{20}$. It may lead to mild elevations in transaminases, which occur in up to $50 \%$ of patients with untreated hyperthyroidism. thyrotoxicosis might also have a direct toxic effect on hepatic tissue. This may interfere with bile transport resulting in cholestasis with hyperthyroidism. In a rat model of thyrotoxicosis, plasmatic and intracellular organoid membranes of hepatocytes in ultrastructure were significant damaged, which has an adverse effect on the functionality of the liver ${ }^{21}$. The right heart failure in long-standing hyperthyroidism can cause passive liver congestion Liver dysfunction may range from mild hyperbilirubinemia, coagulopathy, and hepatomegaly to ascites and liver cirrhosis. Decreased cardiac output may be associated with acute hepatocellular necrosis with marked elevations in serum aminotransferases ${ }^{22}$. Therefore, the guidelines for the management of thyroid storm recommends that treatment of congestive heart failure could contribute to the recovery of normal liver function. which is one of the most common causes of hepatic damage and jaundice ${ }^{13}$.

we suspected the pre-existing chronic liver disease in this patient until the current episode, mainly because she had taken herbal remedies for two years, cases of herb-induced liver injury have been highlighted in many publications. Hyperthyroidism also reasonably consist of chronic liver injury as result of a long period of noncontrolled hyperthyroidism due to her poorly ATD compliance and the paucity of liver function tests.

Graves' hyperthyroidism patients should be rendered euthyroid before RAI therapy. Considering possibility of oral ATD-induced hepatotoxicity, methimazole cream has been admitted to our patient for correcting hyperthyroidism, Thyroxine concentration decreased and normalized after 20 days treatment of methimazole cream. we also adopted therapeutic plasma exchange (TPE) to remove harmful substances, circulating thyroid hormone and bilirubin and to replace coagulation factor and plasma factor, which is a useful adjunct as a bridge to the remedy when hyperbilirubinemia cannot be controlled effectively.

In summary, as observed in our clinical practice and described in the literature, tachycardia and AF, early manifestations of hyperthyroidism indicate significant hemodynamic alterations in cardiovascular system, 
Untreated high-output state and hyperthyroidism can lead to ventricular dilation and persistent tachycardia, further deterioration in hemodynamic can result in chronic heart failure and liver dysfunction even a fatal event. The continuum of disease present the pathophysiology development of Graves' hyperthyroidism, Therefore, we have got some conception that hemodynamic variation is a dominant contributing factor of Graves' hyperthyroidism complication, the prompt identification and effective therapeutics of cardiac manifestations in hyperthyroidism patients is compulsory because the prognosis of it may be improved with the appropriate treatment.

\section{ACKNOWLEDGMENTS}

The entire team of nursing staff and doctors attached to the department, who participated in the care of this patient.

\section{CONFLICT OF INTEREST}

The authors declare that they have no competing interests.

\section{FUNDING}

No financial support was received for this study.

\section{AUTHOR CONTRIBUTIONS}

ZYL and JJW were involved in the diagnosis, management of the patient, proposed the study, writing of the manuscript,contributed equally to this work and should be considered joint first author. YF and JBS are the communicating authors provided expert opinion for revisions, critically revised the manuscript. JZ and JX performed the literature search, gathered and organized information regarding the patient. XYL were involved in production of the diagram, visualization and writing the manuscript.

\section{CONSENT STATEMENT}

A written informed consent was obtained from the patient for publication of identifying data.

\section{ETHICAL APPROVAL}

No approval is required.

\section{DATA AVAILABILITY STATEMENT}

The authors confirm that the data supporting the findings of this research are available within the article.

\section{Reference}

1. Leo S D, Lee S Y and Braverman L E, Hyperthyroidism, Lancet, vol. 388, no. 10047, pp. 906-918, 2016

2. Biondi B, Heart failure and thyroid dysfunction, European Journal of Endocrinology (2012) 167 609-618 2012

3. MALIK R, and HODGSON H, The relationship between the thyroid gland and the liver Q J Med 2002; 95:559-569

4. Brent G. The molecular basis of thyroid hormone action. N Engl J Med. 1994;331:847- 853.

5. Danzi S, Klein I. Thyroid hormone and the cardiovascular system. Minerva Endocrinologica. 2004;29:139 $-150$

6. Resnick LM, Laragh JH. Plasma renin activity in syndromes of thyroid hormone excess and deficiency. Life Sci 1982; 30: 585-6. 
7. Biondi B, Palmieri E A, Lombardi G, et al, Effects of thyroid hormone on cardiac function: the relative importance of heart rate, loading conditions, and myocardial contractility in the regulation of cardiac performance in human hyperthyroidism. J Clin Endocrinol Metab 2002,

8. Brandt F, Green A, Hegedüs L, et al A critical review and meta-analysis of the association between overt hyperthyroidism and mortality. Eur J Endocrinol 2011; 165: 491-7.

9.Klein I, Ojamaa K. Thyrotoxicosis and the heart. Endocrinol Metab Clin North Am 1998;27:51-62.

10.Klein I, Danzi S: Thyroid disease and the heart. Circulation 2007, 116:1725. ttp://dx.doi.org/10.1161/CIRCULATIONAHA.106.678326.

11. Petersen P, Hansen JM. Stroke in thyrotoxicosis with atrial fibrillation. Stroke 1988; 19: 15-8.

12. Akamizu T, Satoh T, Isozaki O, et al. (2012) Diagnostic criteria, clinical features, and incidence of thyroid storm based on nationwide surveys. Thyroid 22: 661-679.

13. Satoh T, Isozaki O, Suzuki A, et al 2016 Guidelines for the management of thyroid storm from The Japan Thyroid Association and Japan Endocrine Society (First edition)

14. Staffurt J S, Gibberd J S, Tang FS: Arterial embolism in thyrotoxicosis with atrial fibrillation. Br Med J 1977, 2:688-690

15. Sawin CT, Geller A, Wolf PA, et al. Low serum thyrotropin concentrations as a risk factor for atrial fibrillation in older persons. N Engl J Med. 1994 Nov 10;331(19):1249-52.

16.Bartalena L, Diagnosis and management of Graves disease: a global overview. Nat Rev Endocrinol 2013; 9: 724-734.

17.Thurnheer R, Jenni R, Russi EW, et al. Hyperthyroidism and pulmonary hypertension. J Intern Med $1997 ; 242: 185-8$.

18.Eleftheraidis D, Fourlas E. Eleftheriadis, N, Hidden hyperthyroidism in a young male patient. Int J Cardiol 2003 Jun;89(2-3):313-4

19. Holmes S. J, Espiner E. A, Richards A M, et al. Renal, endocrine, and hemodynamic effects of human brain natriuretic peptide in normal man. J. Clin. Endocrinol. Metab. 1993, 76, 91-96.

20. Myers J D, Brannon E S, Holland B C. A correlative study of the cardiac output and the hepatic circulation in hyperthyroidism. J Clin Invest1950; 29(8): 1069-1077.

21. Pasyechko N V, Kuleshko I I, Kulchinska V M. et al. Ultrastructural liver changes in the experimental thyrotoxicosis, Pol J Pathol 2017; 68 (2): 144-147

22 Alvarez A M, Mukherjee D, Liver Abnormalities in Cardiac Diseases and Heart Failure. Int J Angiol 2011;20:135-142.

Abbreviation

AF: atrial fibrillation

ATD: anti thyroid drug

BNP: brain natriuretic peptide

CHF: congestive heart failure

CO: cardiac output

DCT: distal convoluted tubule

ECG: Electrocardiogram 
EF: ejection fraction

FFP: fresh frozen plasma

LVDd: left ventricular end-diastolic dimension,

LVEF: left ventricle ejection fraction

MMI: methimazole

PAP: pulmonary arterial pressure

PH: Pulmonary hypertension

RAAS: renin-angiotensin-aldosterone system

RAI: Radioactive Iodine

RVDd: right ventricular end-diastolic dimension

SV: stroke volume

SVR: systemic vascular resistance

TPE: therapeutic plasma exchange

TSH: thyroid stimulating hormone

\section{Hosted file}

Fig 1.pptx available at https://authorea.com/users/437578/articles/539114-hemodynamicvariation-is-a-dominant-contributing-factor-of-graves-hyperthyroidism-complicationheart-failure-and-fatal-liver-disfunction-a-case-report-and-analysis

\section{Hosted file}

Fig 2.pptx available at https://authorea.com/users/437578/articles/539114-hemodynamicvariation-is-a-dominant-contributing-factor-of-graves-hyperthyroidism-complicationheart-failure-and-fatal-liver-disfunction-a-case-report-and-analysis

\section{Hosted file}

Fig 3.pptx available at https://authorea.com/users/437578/articles/539114-hemodynamicvariation-is-a-dominant-contributing-factor-of-graves-hyperthyroidism-complicationheart-failure-and-fatal-liver-disfunction-a-case-report-and-analysis 